\title{
A THING SPEAK IOT BASED VIBRATION MEASUREMENT AND MONITORING SYSTEM USING AN ACCELEROMETER SENSOR
}

\author{
Shubhangi Kumari \\ Department of ECE \\ MIT-WPU, PUNE, MAHARASTRA, INDIA
}

\author{
Rohit Raj \\ Department of ECE \\ MIT-WPU, PUNE, MAHARASTRA, INDIA
}

\author{
Rajkumar Komati \\ Department of ECE \\ MIT-WPU, PUNE, MAHARASTRA, INDIA
}

\begin{abstract}
Thing Speak IoT on a Real-Time Vibration measurement System using the accelerometer Sensor. Many systems have been designed previously but some lack systems are identified where it does not provide adaptively connections and alert to web pages on logging data collections. Thus, understanding the previous system model is important to compare the importance of new build parameters in designing the new system. An evaluation of the current model, hardware, and software is important before a new architecture is developed. This research has developed a prototype system to monitor and measure the vibration of a system remotely and setting up threshold and alert accordingly. The designed system assisted with an internet as vibration monitoring system for the machines. Research methods consist of two parts involved hardware and software development. The hardware development covers the connections of the accelerometer sensor and the software involved in constructed coding using the $\mathrm{C}$ language program. The program then is compiled and uploaded into the Node MCU to display the acceleration of the system. An open-source Internet of Things called Thing Speak is used as a platform to retrieve and display the collected data. Real-time monitoring can be accessed through Smartphone and web applications. This study has been considered successfully implemented and it is a significant study that performs on new IoT platforms and adaptively ready to monitor a vibration remotely.
\end{abstract}

Keyword- Accelerometer Sensor, Internet of Things, Thing Speak, Real-Time.

\section{INTRODUCTION}

The term IoT means 'Internet of Things', which was coined by Kevin Ashton in 1999 [1]. It is a budding technology that plays a major role in today's life to Interconnect devices and the internet in a network, which in turn enables them to send and receive data [2].

In today's automated industrial era every large or smallscale industries are completely dependent on machines and if machines get failed it causes a severe loss to an industry sometimes also causes collapse of industry and in failure of any machine component breakout, misalignment, motor failure, etc. play an important role that causes an unusual occurrence of vibration in the machinery. Vibration analysis is one of the most efficient and successful techniques for fault diagnosis of rotating machinery [3]. In this project, we are going to read the unusual vibration that occurred in any part of the machinery and also try to find out the reason behind it using a wireless vibration measurement system using an accelerometer.

Previous research has presented that many vibration monitoring systems are monitored using a data logger that misses some data and doesn't give perfect results or alert if something happens. Nowadays, the vibration monitoring system is widely used in various processes like motors, mobile phones, automotive industries, earth quake detecting systems, and other industries where data collections are analyzed and evaluated. Todays, most research are developing the Internet of Things (IoT) research where the network of physical objects devices, II. Proposed Algorithm

vehicles, buildings, and other items embedded with electronics, software, sensors, and network connectivity. IoT development enabled its sensors to collect data and the controller played the brain of central connectivity. Arduino controller are mostly used which its makes development easier especially for small IoT research project [4]. Arduino and Node MCU controllers are mostly used which makes its development easier especially for small IoT research projects. Node MCU is used in the electronic project which is divided into two parts. The first part is the hardware program and the second part is the software part. The second part which is an 
important part of Node MCU is the part that has been used to upload coding from a computer into the board. Node MCU and Arduino does not

need the other components to complete the upload coding which is Node MCU having their USB port. Many IoT systems development used the $\mathrm{C}++$ language for the interface configuration. Besides Node MCU some research used ESPresso Lite V2.0 which acts as the internet board for the project to connect the software to hardware. ESPresso Lite V2.0 uses the ESP8266 Wi-Fi platform as a Wi-Fi module.

The ESP8266/NODE MCU is prepared to offload all Wi-Fi networking capacities from another application processor. ESP8266 has low power consumption with a few exclusive advanced features. This internet board allows anyone to learn, experiment and build their IoT projects easily, quickly and remotely access [5]. The core hardware inside NODE MCU is ESP8266 with the development of Information Technology, network, and automatic control technology. Adaptive IoT vibration monitoring system used one type of acceleration recorder that monitors vibration in a server room and displays the current acceleration on the website through a web server and Smartphone application. Thing Speak web is an example of a website using open-source data platform of IoT [6]. A device that connected with Thing Speak was advertised and displays its result on the Thing Speak website and the data is saved in private or as public data.

Besides, the data that are saved using Thing Speak, data also is taken sampled to be analyzed. Thing Speak gave the online text editor to perform data analysis and real-time data collection [7]. This paper presents a Thing Speak IoT on Real Vibration measurement and Monitoring System which measured the system acceleration using an accelerometer sensor. The monitoring system was applied in industry to obtain the machine conditions. The problem occurs of certain high vibration need to measure in real-time where data is needed to analyze often when needed. Cases to keep a good and safe environment need by some industries to give a safe working environment to their workers and avoid sudden failure of the system.

\section{LITERATUREREVIEW}

\section{Existing problem}

Maintenance of equipment means using a method to prevent equipment failure and keep them operational. Maintenance costs are a significant part of the total costs of operation for all factories and manufacturing plants. Maintenance costs can be from $15 \%$ to $60 \%$ [8] even $70 \%$ [9] depending on the industry of total costs. Maintenance management techniques can be classified into three key categories [10] that are as follows in order of efficiency [11]:
a.

\begin{abstract}
Run-to-Failure (R2F) or Corrective Maintenance - Maintenance procedures are carried out only after failures have occurred.
\end{abstract}

b. Preventive or Planned Maintenance (PvM) Maintenance is performed based on time or process iterations according to a planned schedule.

c. Predictive Maintenance (PdM) - Maintenance is conducted based on an assessment of the health.

Status of the equipment that comes from monitoring.

Monitoring is the key element of PdM [8], in other words, PdM can be considered as condition based PvM. The maintenance program, particularly the PdM approach, that requires constant monitoring of the equipment, is often applied for an important equipment category called rotating machinery [8], which includes motors, pumps, fans, turbines, gearboxes, etc. [8].The IoT is the network that connects things together. These things can be computers, smart phones, people, data, processes, and physical objects such as machines, devices, and appliances [12]. IoT can integrate various manufacturing devices in order to sense, identify, process,

\section{Proposed solution}

The solution that can address these problems is to make the equipment smart. Equipment must be able to announce its status at any time and this capability leads us to the concept of the Internet of Things (IoT). Communicate, operate and network. This deeply integrated intelligent cyber physical environment brings so many opportunities and values for manufacturing businesses [13]. Using IoT inside the industry is called industrial Internet of things (IIoT). This term refers to the industrial subset of the IoT. IIoT in manufacturing could make so much value that it will finally head to the Fourth Industrial Revolution or Industry 4.0 [14]

\section{AIM AND OBJECTIVES OF PROJECT}

To overcome the drawback, that is the missing knowledge between sampling sessions, there ought to be

an answer which will convert sectional sampling to continuous sampling or knowledge streaming. The second drawback is human error throughout sampling. The technician might place the sampling device at completely different points at different sampling sessions and procure different results. The sampling ought to be done mechanically to forestall human errors; like mistakes in reading or inserting numbers. succeeding drawback is sampling at completely different conditions of the instrumentation in terms of load, which suggests that sampling could also be performed at a time of utmost load and after be performed at a lower load, which can create the results extraordinary. This drawback is going to be resolved by resolution the primary drawback; as a result of if the sectional sampling converts to an information stream, the instrumentation knowledge is going to be sampled in the least times and it'll be attainable to look at the information at a such time. Another drawback is that the inconvenience of some 
instrumentation for human. to unravel the matter, we'd like to appear for an answer that doesn't need the technician to be at the situation of the instrumentation for sampling.

\section{Problem Statement}

The typical method of vibration analysis in factories is that condition observation technician goes into the mill and measures the vibration of machines employing a knowledge logger; then, he/she investigates the gathered knowledge and in keeping with the familiar patterns of assorted attainable defects which will occur detects the faults. Concerning the diagnosing of severity and sort of faults, he/she announces the requirement for repair or replacement of the instrumentation.

This methodology has some shortcomings such as:

- Human error in sampling.

-Missing knowledge between completely different knowledge sampling sessions which can embody necessary data regarding faults.

- Lack of access to some instrumentation for human.

\section{METHODOLOGY}

\section{A. Flowchart}

Figure1 shows a project of Vibration measurement and monitoring System supported IoT. With the help of this system, we can analyze the vibration of system using web packages and hardware. Web board NODE MCU, acceleration sensor (adxl335), adc115 ADC converter, led and GPS-module are used as the hardware of the project. The system used the website Thing Speak, Smartphone app, MATLAB and telemetry viewer application as the software of the project.

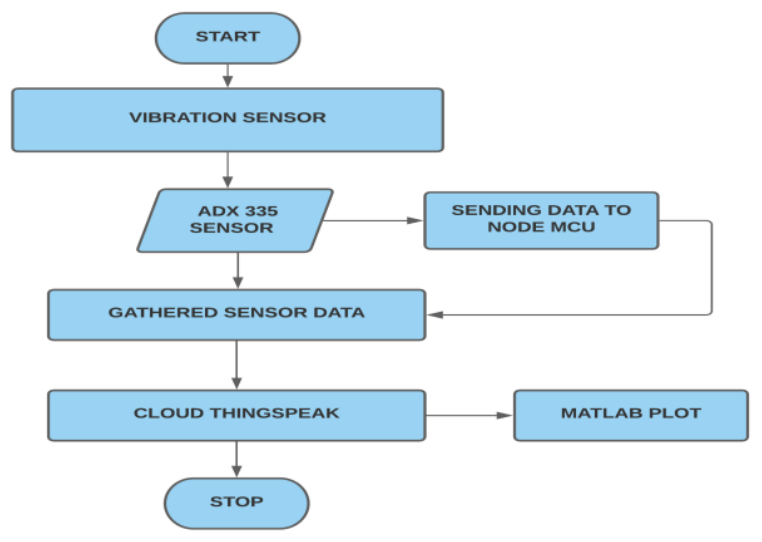

Fig.1.System'sflowchart

\section{A. Block Diagram}

Figure 2shows a purposeful diagram of the system. Different parameter was utilized in this project to sense acceleration of system using accelerometer sensor ( $\operatorname{adxl335)}$, the info gathered from the sensor was sent to the Microcontroller of the system that is Node MCU. the microcontroller has processed the data and transmitted the data via Wi-Fi using http protocol to cloud that the user will see information through the web site and Smartphone application. aside from that, the data is also stored into a csv file using open-source software telemetry viewer and then the csv file is imported into MATLAB for FFT plotting.

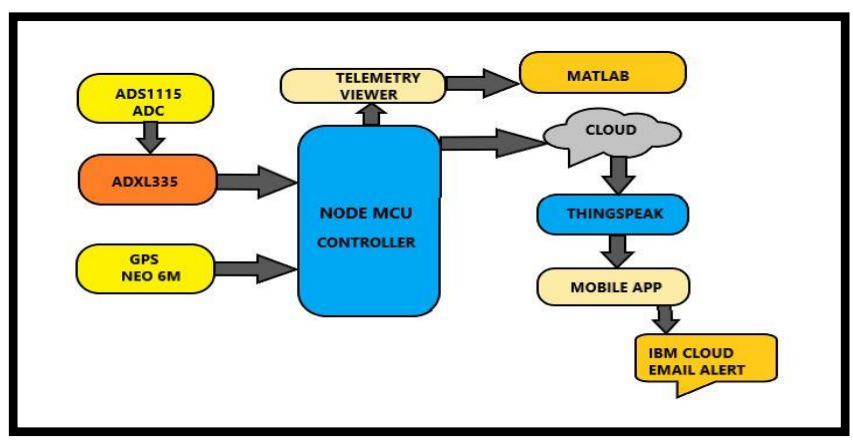

Fig.2.System'sBlock diagram

This IoT primarily based project has four sections. First, Vibration sensors can sense and analyze the acceleration data. Second, Node MCU will be acting as bridge between virtual (Internet) and physical (Vibration) world by connecting it to the internet. Third, the data received from measuring device are displayed over an online Application or App (Frontend) to observe and for correct understanding of the information. Data is updated each $15 \mathrm{sec}$ on Thing Speak. And for storing the data we have open-source website THING SPEAK (Backend) and TELEMETRY viewer for offline storage. Thing Speak server is a open source platform and API for the web of Things that permits to gather, store, analyze, visualize, and act on data from sensors, and at last, For graphical illustration like acceleration amplitude, frequency plot etc. we are going to $b$ use MATLAB software by Math Works. MATLAB is also compatible with Thing Speak platform.

Figure 3 shows the data from GPS module that consists of longitude and latitude of the system location is displayed over Thing Speak using http protocol, Node MCU as controller connected to Neo 6M GPS module. The figureshows Neo 6M collects the latitude and longitude data as shortly as power is on the system started operating. The data is updated each fifteen sec over thing speak platform. 


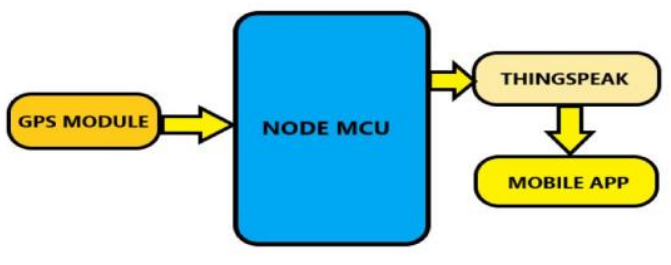

Fig.3.System with GPS module

Figure 4 shows data from sensor is stored using telemetry vieweropen-source platform in a csv file. The software additionally plots live time domain plot of the data receiving from Node MCU through COM port connected. once the data is stored into a csv file by the telemetry viewer, the data will be imported to MATLAB for frequency and time domain analysis of vibration. This FFT graph tells us the changing nature of vibration, for a system frequency is fixed if the frequency is changing from natural value to some other values that mean there is some problem in system.

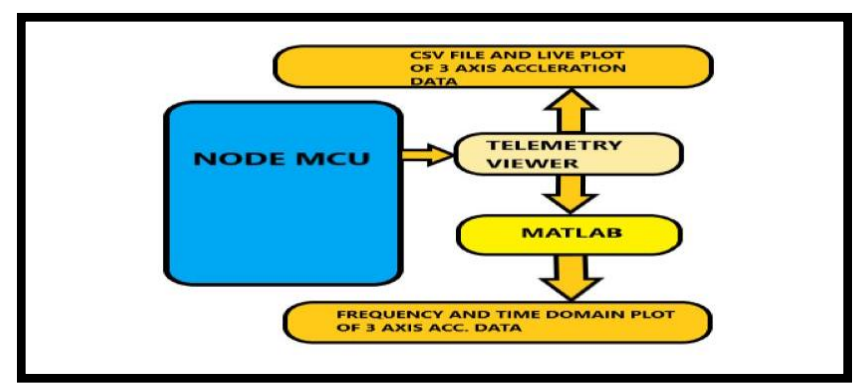

Fig.4.Telemetry and MATLAB connection diagram

\section{RESULTANDANALYSIS}

The system was successfully developed as the sensor may gather the information from the system to be displayed at website and developed mobile application. Figure 5 shows the app development part, which is developed using MIT app inventor specially for this project and the data collection done by the adx1335 sensor and displayed on app. As the system is turned on the sensor starts detecting and collects the information from the system. The data updated in every $15 \mathrm{sec}$ of interval over mobile application.

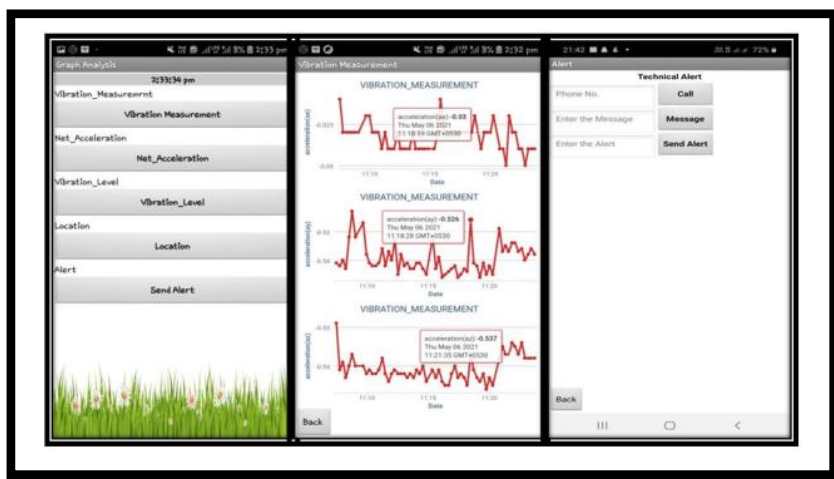

Fig.5. Mobile Application view

Figure 6 show the data collected from the website which is from the Thing Speak website using http protocol. The data collection at the Thing Speak website is represented in a graph against the day for each time the data are updated on that day. Even though the graph was shown in a day, the data may show the record of each data entry form that day by pointing mouse pointer on that day on the graph.

The graphs in figure display the data along 3 axes of adx1335 and plotted in different fields, the data from gps module is also plotted here.

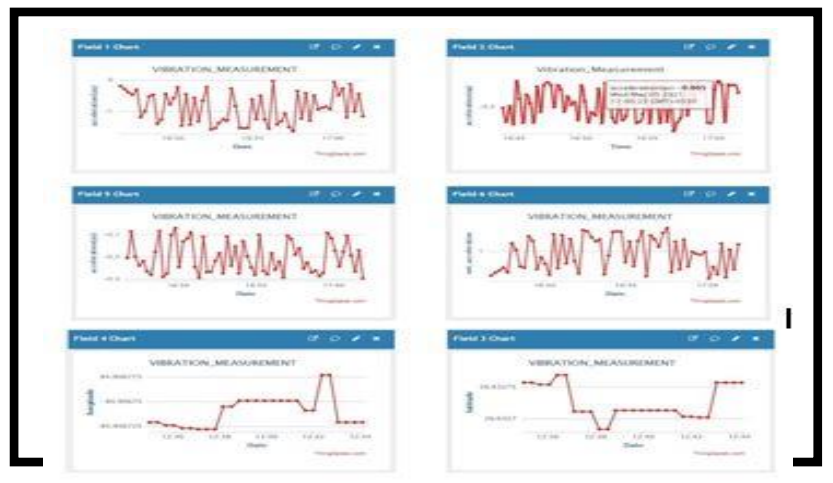

Fig.6. Thing Speak view with 3 -axis data.

Figure 7 and Figure 8 shows the data collection from the telemetry viewer and stored in a csv file and Real-time plotting of data received from Node MCU using COM port.

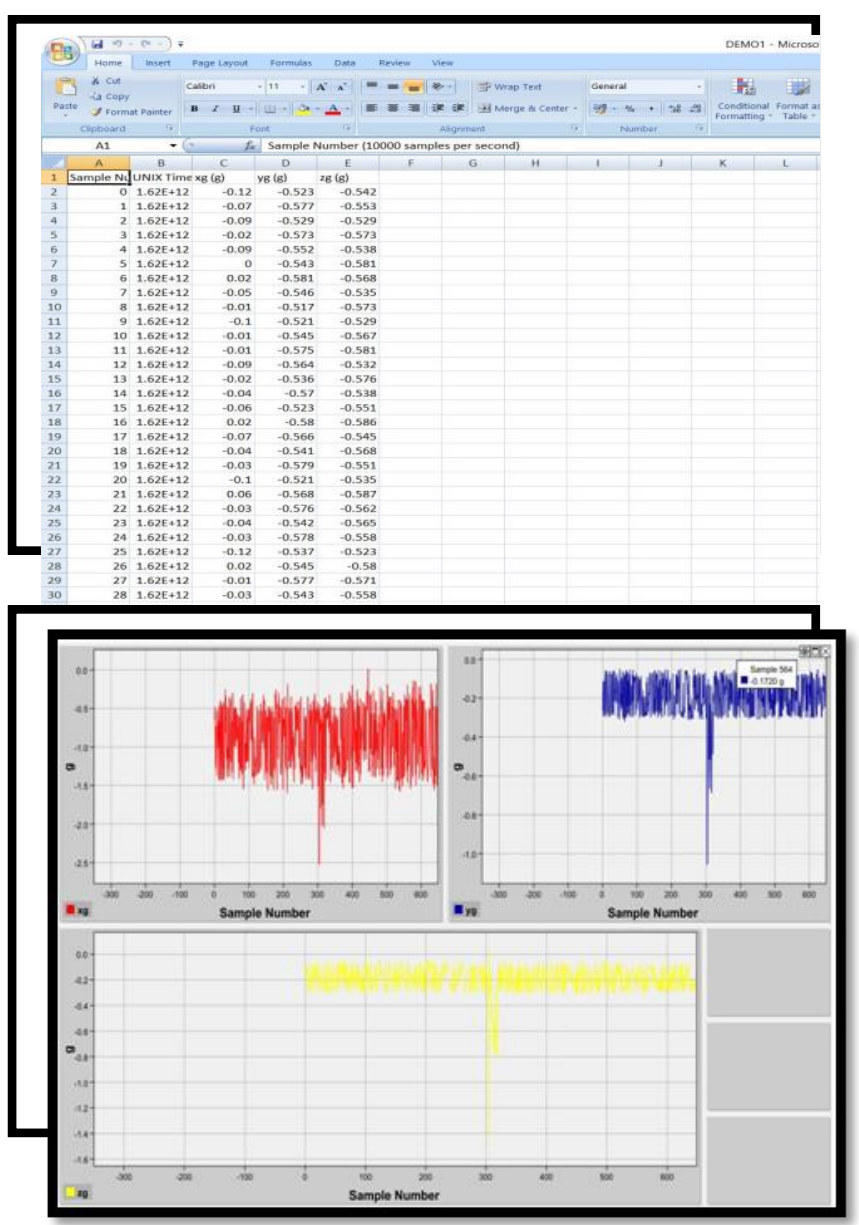


Fig.8. Telemetry viewer 3-axis X, Y, Z live plot.

figure 9 shows the graph plotted by MATLAB over x-axis. The plotted graph contains data in time domain and data in frequency domain to measure the frequency of vibration in hertz and time in second.

The $\mathrm{x}$-axis of FFT graph displays amplitude of acceleration and y axis displays the frequency at different amplitudes. The highest spike in FFT graph gives the frequency of system to which the hardware is connected.

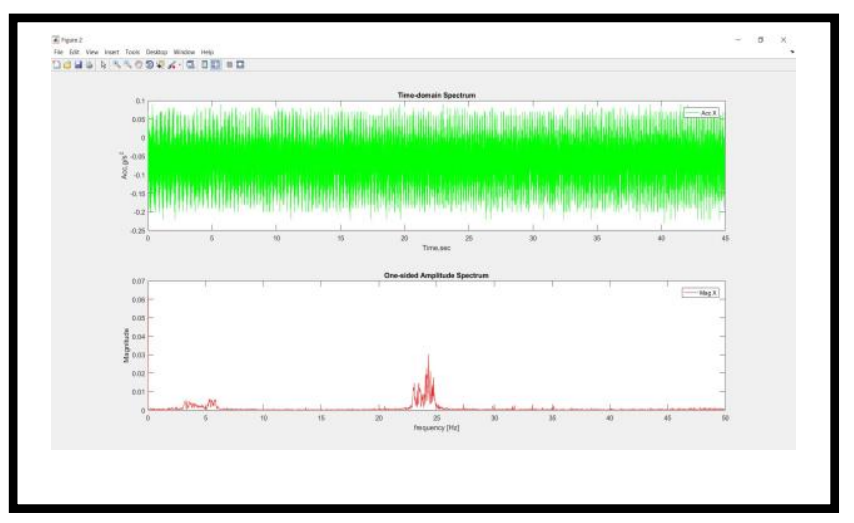

Fig.9. MATLAB plot for frequency

figure 10 and figure 11 shows the development of alert system using IBM cloud and node red for email SMS alert in case of high vibration or change in vibration detected. If the value of acceleration is above threshold the mobile application will send an alert email to the authorized person to take action.

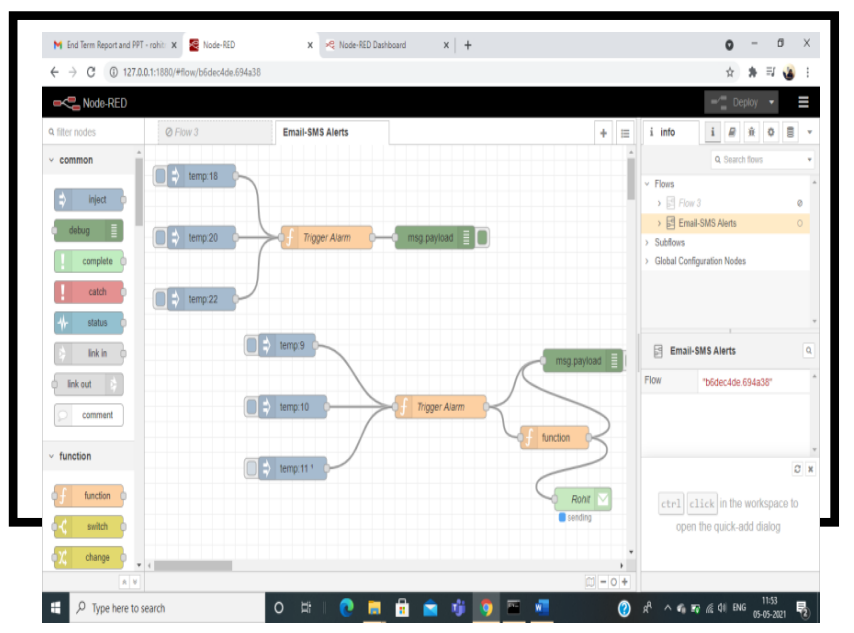

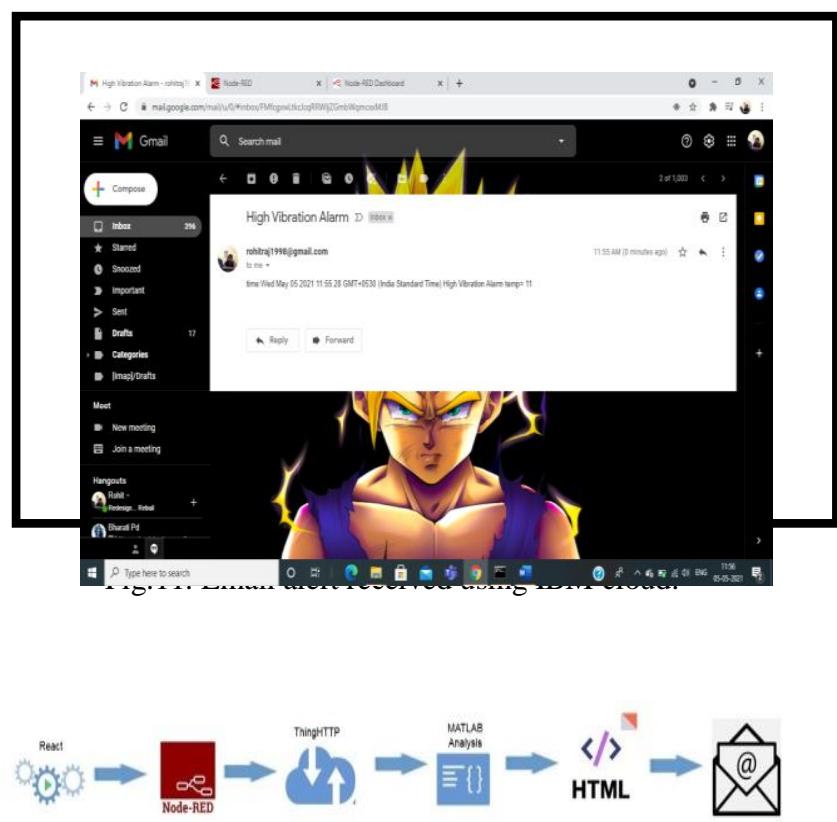

figure 12 shows the hardware part of the development of the system. The hardware part is consisting of components like adx1335, adc1115, Neo 6M GPS module, LEDs and PCB. In case of vibration measurement, the hardware is attached to the system who's vibration we need to check and then the data sensed by adxl335 is displayed over serial monitor and also updated to website, mobile application and csv file for further analysis.

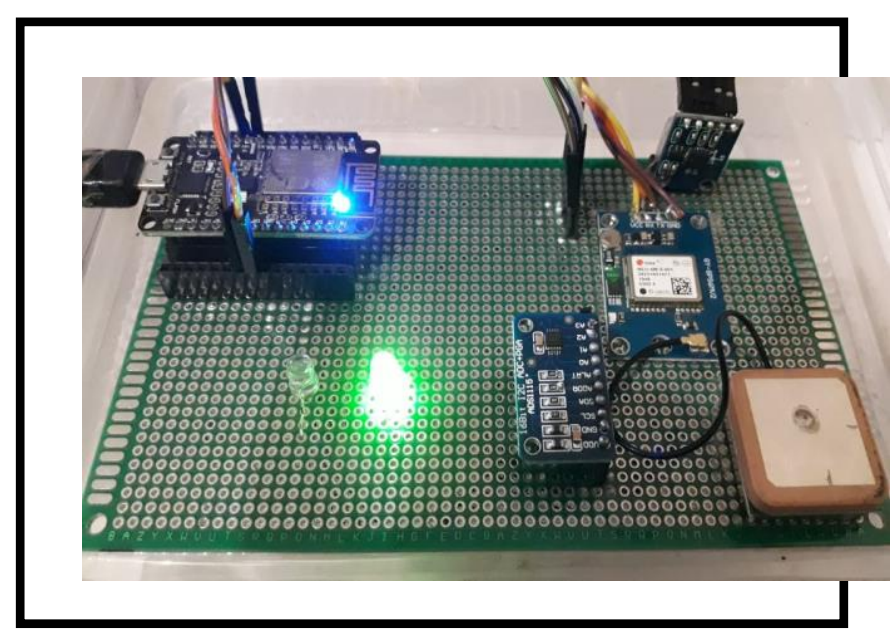

Fig.12. Working hardware of system 


\begin{tabular}{|l|l|}
\hline \multicolumn{2}{|l|}{ ADXL335 MODULE SPECIFICATION } \\
\hline INTERFACE: & $3 \mathrm{~V} 3 / 5 \mathrm{~V}$ Microcontroller \\
\hline VOLTAGE REQUIREMENT: & $3-6 \mathrm{~V}$ DC \\
\hline OUTPUT FORMAT: & ANALOG OUTPUT \\
\hline MEASURING RANGE: & $+-3 \mathrm{~g}$ \\
\hline MEASURING VALUES $(-3$ to+3): & $\begin{array}{l}\mathrm{X}(-274 \text { to }+325) \\
\mathrm{Y}(-275 \text { to }+330) \\
\mathrm{Z}(-275 \text { to }+310)\end{array}$ \\
\hline
\end{tabular}

TABLE1.Specification of accelerometer sensor adxl335

\begin{tabular}{|l|l|}
\hline \multicolumn{2}{|l|}{ NODE MCU(ESP8266-12F) SPECIFICATOION } \\
\hline OPERATING VOLTAGE: & $3.3 \mathrm{~V}$ \\
\hline DIGITIAL I/O PINS: & 12 \\
\hline ANALOG INPUT PINS: & 1 \\
\hline CLOCK SPEED: & $80 \mathrm{MHZ} / 160 \mathrm{MHZ}$ \\
\hline FLASH: & $4 \mathrm{M}$ BYTES \\
\hline WIDTH: & $29.1 \mathrm{~mm}$ \\
\hline COST: & $₹ 575.00$ \\
\hline
\end{tabular}

TABLE 2.Specification of controller NODE MCU.

\begin{tabular}{|l|l|}
\hline \multicolumn{2}{|l|}{ NEO 6M GPS MOSULE SPECIFICATION } \\
\hline RECEIVER TYPE: & 50 channels, GPS L1(1575.42Mhz) \\
\hline OPERATING VOLTAGE: & $2.7 \mathrm{~V} \sim 3.6 \mathrm{~V}$ \\
\hline OPERATING CURRENT: & $45 \mathrm{~mA}$ \\
\hline SERIAL BAUD RATE: & $4800-230400(9600$ default) \\
\hline CAPTURE TIME: & Cool start: 27 sHot start: $1 \mathrm{~s}$ \\
\hline $\begin{array}{l}\text { HORIZONTAL POSITION } \\
\text { ACCURACY: }\end{array}$ & $2.5 \mathrm{~m}$ \\
\hline COST: & $₹ 715.00$ \\
\hline
\end{tabular}

TABLE3.Specification of GPS Module NEO 6_M

\section{CONCLUSION}

In this paper, we demonstrated the potentials of IoT for smart maintenance. We explained how IoT can help the condition monitoring and fault diagnosis of rotating machinery using vibration analysis. Further, vibration sensing has been investigated and the new generation of MEMS accelerometers has been introduced. We discussed the superiority of MEMS accelerometers on legacy piezoelectric accelerometers and their importance in the future of smart maintenance. We proposed to make rotating equipment to IoT enabled devices by adding hardware to them. According to the requirements, the hardware was designed and developed. This hardware can measure vibrations with $2.5 \mathrm{kHz}$ sampling rate in the range of $\pm 3 \mathrm{~g}$ and send them in real-time to a specified server on the cloud. This way the machine will be able to send its vibration status to the server and the maintenance engineers will be able to monitor it anytime, anywhere. The developed hardware has been evaluated by comparing the results of online and local data measurements at the same time. The results of the evaluation have been proved to be very precise. This paper will prove the concept of using IoT enabled sensors in predictive maintenance to overcome some gaps in the commonly used methods of condition monitoring of rotating machinery. The results can be extended to a larger scope of equipment, faults, and parameters.

\begin{tabular}{|l|l|}
\hline Project/Description & $\begin{array}{l}\text { IoT Based Vibration } \\
\text { measurement and } \\
\text { monitoring system. }\end{array}$ \\
\hline Concept & $\begin{array}{l}\text { Monitor and measures } \\
\text { the acceleration of a } \\
\text { system in terms of g } \\
\text { which is also a unit to } \\
\text { measure vibration of a } \\
\text { system. }\end{array}$ \\
\hline Device & NODE MCU ESP8266- \\
& $12 \mathrm{~F}$ \\
\hline Software & Arduino platform \\
& interface,Thing Speak \\
& website, MIT app \\
inventor, MATLAB, \\
IBM cloud, Telemetry \\
viewer.
\end{tabular}

TABLE4. Parameters of IoT Based system.

\section{REFERENCES}

[1] Hopalı Egemen, Vayvay Özalp, (2018) "Internet of Things (IoT) and its Challenges for Usability in Developing

Countries" International Journal of Innovation Engineering and Science Research, Vol. 2.

[2] Alfarsi Ghaliya, Tawafak Ragad M, Alsidiri Abir, Jabbar Jasiya, Malik Sohail Iqbal, Alsinani Maryam,(2019), “Using Cisco Packet Tracer to simulate Smart Home", Vol. 8.

[3] Chaudhury, Subimal Bikash, Sengupta Mainak, and Mukherjee Kaushik. (2014),"Vibration monitoring of rotating machines using MEMS accelerometer." International Journal of Scientific Engineering and Research 2, pp. 9-11. 
[4] Rahman R. A., Aziz N. S. A., Kassim M., and Yusof M. I., (2017) "IoT-based personal health care monitoring device for diabetic patients," in ISCAIE 2017 - 2017 IEEE Symposium on Computer Applications and Industrial Electronics, pp. 168-173.

[5] Yahaya C. K. H. C. K., Ismail M. N., and Kassim M.,(2010), "A study on automated, speech and remote temperature monitoring for modeling Web based temperature monitoring system," in 2010 2nd International Conference on Education Technology and Computer, pp. V5-229-V5-233.

[6] Maureira M. A. G., Oldenhof D., and Teernstra L., (2011) "ThingSpeak-an API and Web Service for the Internet of Things," World Wide Web.

[7] Aghaei S., Nematbakhsh M. A., and Farsani H. K., (2012),"Evolution of the world wide web: From WEB 1.0 TO WEB 4.0," International Journal of Web \& Semantic Technology, vol. 3, pp. 1-10.

[8] Mobley, Keith R..,(2002), An introduction to predictive maintenance. Elsevier.

[9] Dhillon, Balbir S., (2006), Maintainability, maintenance, and reliability for engineers. CRC press.

[10] Susto, Antonio Gian, Schirru Andrea, Pampuri Simone, McLoone Seán, and Beghi Alessandro Beghi. (2015), "Machine learning for predictive maintenance: A multiple classifier approach." IEEE Transactions on Industrial Informatics 11, no. 3:812-820.

[11] Susto, Antonio Gian, Beghi Alessandro, and Luca Cristina De., (2012), "A predictive maintenance system for epitaxy processes based on filtering and prediction techniques." IEEE Transactions on Semiconductor Manufacturing 25, no. 4:638-649.

[12] Wang, Chen, Vo Hoang Tam, and Ni Peng ,( 2015), "An IoT application for fault diagnosis and prediction." In Data Science and Data Intensive Systems (DSDIS), 2015 IEEE International Conference on, pp. 726-731.

[13] Yang, Chen, Shen Weiming, and Wang Xianbin,(2018), "The internet of things in manufacturing: Key issues and potential applications." IEEE Systems, Man, and Cybernetics Magazine 4, no. 1: 6-15.

[14] Daugherty, Paul, Banerjee Prith, Negm Walid, and Alter Allan E.,(2015), "Driving unconventional growth through the industrial internet of things." accenture technology. 